\title{
Hybrid Swarm Algorithm for Multiobjective Optimal Power Flow Problem
}

\author{
K. Rajalashmi ${ }^{1}$, S. U. Prabha ${ }^{2}$ \\ ${ }^{1}$ Department of Electrical and Electronics Engineering, Bannari Amman Institute of Technology, Sathyamangalam, India \\ ${ }^{2}$ Department of Electrical and Electronics Engineering, Sri Ramakrishna Engineering College, Coimbatore, India \\ Email: rajalashmik@bitsathy.ac.in
}

How to cite this paper: Rajalashmi, K. and Prabha, S.U. (2016) Hybrid Swarm Algorithm for Multiobjective Optimal Power Flow Problem. Circuits and Systems, 7, 35893603.

http://dx.doi.org/10.4236/cs.2016.711304

Received: May 17, 2016

Accepted: May 28, 2016

Published: September 8, 2016

Copyright $\odot 2016$ by authors and Scientific Research Publishing Inc. This work is licensed under the Creative Commons Attribution International License (CC BY 4.0).

http://creativecommons.org/licenses/by/4.0/

\begin{abstract}
Optimal power flow problem plays a major role in the operation and planning of power systems. It assists in acquiring the optimized solution for the optimal power flow problem. It consists of several objective functions and constraints. This paper solves the multiobjective optimal power flow problem using a new hybrid technique by combining the particle swarm optimization and ant colony optimization. This hybrid method overcomes the drawback in local search such as stagnation and premature convergence and also enhances the global search with chemical communication signal. The best results are extracted using fuzzy approach from the hybrid algorithm solution. These methods have been examined with the power flow objectives such as cost, loss and voltage stability index by individuals and multiobjective functions. The proposed algorithms applied to IEEE 30 and IEEE 118-bus test system and the results are analyzed and validated. The proposed algorithm results record the best compromised solution with minimum execution time compared with the particle swarm optimization.
\end{abstract}

\section{Keywords}

Multiobjective, OPF, Optimization, Hybrid

\section{Introduction}

The power system is an interconnected electric network, which has generating plant and loads that are connected through transmission and distribution networks. It is a complicated network and it has many objectives to be solved. The reliable result of the objectives is obtained using optimal power flow. Optimized solution for the objective functions is attained by satisfying the power flow equations and constraints of the entire power system network. Various control variables are also influenced to achieve an 
optimal solution. Many conventional techniques are applied to solve the optimal power flow problem. They are quadratic programming [1], interior programming [2], linear programming [3], gradient method [4] and newton's method [5]. The drawback of these methods is that it is a local solver only. The solutions from these classical methods are not the most reasonable for nonlinear, discrete and continuous functions with constraints.

In order to overcome the drawbacks of classical methods, many computational methods are used to solve the optimal power flow problem. Particle Swarm Optimization (PSO) [6] inspires the school of fishes and a flock of birds and trapped in local optima. Genetic algorithm [7] [8] solves the optimal power flow problem using a random selection of starting values of voltage angles, but it has no control over global and local investigation. Evolutionary programming [9] algorithm solves the problem by enhancing the gradient of the function, and it has the problem of long computational time. Tabu search [10] solves the optimal power flow problem which is more composite for classical method. These manuscripts give the optimal solution for individual objective. Conversely the real world problem needs a concurrent optimal solution for all the objectives.

Multiobjective optimal power flow problem is solved by differential evolution, for active and reactive power dispatch [11] and fuel cost, voltage profile, and voltage stability enhancement [12]. The drawback of this technique is that the convergence time is more. Enhanced genetic algorithm [13] solves the problem with the combination of new developed quadratic load flow and its demerit is that it gets trapped in local optima. Self adaptive evolutionary programming [14] combines the evolutionary programming and random search technique and it has the problem of long computational time. As particle swarm optimization is improved [15], the particles avoid being trapped in local optima. Harmony search algorithm [16] inspires the analogy with music improvisation which has solved the problem with slow convergence. These techniques solve the problem with the long computation time and being trapped in local optima.

In the weighted sum method [17], different objectives are formatted into a single function. In this method, the demerit is to run the problem with multiple time to desire the optimal solution. Voltage security costs [18] are optimized using a penalty method which has the drawback of deciding the suitable penalty. The importance of each objective is not preserved while merging all the objective function into a single objective function. In order to maintain the significance of each objective pareto optimal method is adopted.

In the PSO algorithm, if the particles are small, local minimum problem will occur and the outcome could be evaluated in multiple runs. If the particles are too large, the global solution is obtained in each run and it reduces the speed of the algorithm. In order to overcome these difficulties and to increase the efficiency of PSO in this paper, a new hybrid technique technique is proposed by combining the particle swarm optimization and ant colony optimization with chemical communication signal.

In this paper cost, loss and Voltage Stability Index (VSI) objectives are analyzed using enhanced PSO with the multiobjective optimal power flow by pareto optimal method. The multiobjective optimization of these objectives is practiced through three 
cases. In the first case, all the objective functions are simulated individually. In the second case, two objectives are considered simultaneously for optimization such as cost-loss, cost-VSI, loss-VSI and in the third case three objectives are considered concurrently as cost-loss-VSI. These objectives are optimized using the proposed technique with the IEEE 30 and IEEE 118-bus test system. A short introduction of an optimal power flow system has been presented in this section. This paper is planned as a tag on the section. In Section 2, the optimal power flow problem is formulated and discussed. Section 3 explains the central concepts of PSO, Ant colony Optimization (ACO) and Hybrid Swarm Optimization. Section 4 presents the simulated results with discussions of PSO, ACO and hybrid PSO. The conclusion is dealt in the Section 5.

\section{Problem Formulation for OPF Solution}

In this paper the cost, transmission loss, voltage stability index are optimized, which is satisfying equality and voltage, generator, shunt VAR and transformer constraints.

\subsection{Problem Objectives}

1) Minimization of Fuel Cost:

This objective is to minimize the cost. The total fuel cost can be expressed as

$$
\begin{aligned}
& \text { Minimization } \sum_{i=1}^{n} F_{i}\left(P_{i}\right) \\
& F_{i}\left(P_{i}\right)=a_{i} P_{i}^{2}+b_{i} P_{i}+c_{i} \\
& P_{i}^{\min } \leq P_{i} \leq P_{i}^{\max }
\end{aligned}
$$

$a_{i} b_{p}, c_{i}$ is the cost coefficients of the ith generator, $P_{i}$ is the real power output of the ith generator.

2) Minimization of Transmission Loss:

This objective is to minimize the real power transmission loss. The transmission loss can be stated as

$$
T L=\sum_{k=1}^{n} g_{k}\left[V_{i}^{2}-V_{j}^{2}-\left(2 V_{i} V_{j} \cos \left(\theta_{i}-\theta_{j}\right)\right)\right]
$$

$V_{i}$ is the voltage at the ith line, $V_{j}$ is the voltage at the jth line, $g_{k}$ conductance.

3) Minimization of Voltage Stability Index:

This objective is to minimize the voltage stability index. The voltage level ought to maintain below critical level. If the voltage level greater than the critical limit the entire system becomes unstable. The fast voltage stability index expressed as

$$
F V S I_{i j}=4 Z_{i j}^{2} Q_{j} / V_{i}^{2} X_{i j}
$$

$Z_{i j}$ is the impedance of line between $i$ and $j, \quad X_{i j}$ is the reactance of line between $i$ and $j, V_{i}$ is the sending end voltage, $Q_{i}$ is the receiving end reactive power.

\subsection{Problem Constraints}

1) Voltage Constraint: 
The bus voltage maintained the maximum and minimum level. The voltage limit conveyed as

$$
V_{i}^{\min } \leq V_{i} \leq V_{i}^{\max }
$$

$V_{i}^{\min }, V_{i}^{\max }$ Minimum, maximum values of voltage magnitudes at bus $i$.

2) Generation Constraints:

The real and reactive power of generation sustained the maximum and minimum limit. The generation level of real and reactive power is expressed as

$$
\begin{gathered}
P_{G i}^{\min } \leq P_{i} \leq P_{G i}^{\max } \\
Q_{G i}^{\min } \leq Q_{i} \leq Q_{G i}^{\max }
\end{gathered}
$$

$P_{G i}^{\min }, P_{G i}^{\max }$ Minimum, maximum values of real power allowed at bus $i$.

$Q_{G i}^{\min }, Q_{G i}^{\max }$ Minimum, maximum values of reactive power allowed at bus $i$.

3) Transformer Constraints:

The transformer tap setting values are maintained within the maximum and minimum level. The transformer tap setting limit is expressed as

$$
T_{i}^{\min } \leq T_{i} \leq T_{i}^{\max }
$$

$T_{i}^{\min }, T_{i}^{\max }$ Minimum, maximum values of transformer tap settings allowed at bus $i$.

4) Shunt VAR Constraints:

The shunt VAR restricted the maximum and minimum level. The shunt VAR limit is stated as

$$
Q_{C i}^{\min } \leq Q_{i} \leq Q_{c i}^{\max }
$$

$Q_{C i}^{\min }, Q_{c i}^{\max }$ minimum, maximum values of Shunt VAR allowed at bus $i$.

5) Equality Constraints:

The equality constraints are expressed as

$$
\begin{aligned}
& P_{G i}-P_{D i}-V_{i} \sum_{j=1}^{n} V_{j}\left[G_{i j} \cos \left(\delta_{i}-\delta_{j}\right)+B_{i j} \sin \left(\delta_{i}-\delta_{j}\right)\right]=0 \\
& Q_{G i}-Q_{D i}-V_{i} \sum_{j=1}^{n} V_{j}\left[G_{i j} \cos \left(\delta_{i}-\delta_{j}\right)+B_{i j} \sin \left(\delta_{i}-\delta_{j}\right)\right]=0
\end{aligned}
$$

$n$ : Number of buses.

$P_{G i} Q_{G i}$ Real, reactive power generation at bus $i$.

$P_{D i} Q_{D i}$ Real, reactive load demand at bus $i$.

$V_{p}, V_{j}$ Voltage magnitudes at bus $i$ and $j$ respectively.

$G_{i j} B_{i j}$ Transfer conductance and susceptance between bus $i$ and $j$ respectively.

$\delta_{p}, \delta_{j}$ Voltage angle at bus $i$ and $j$ respectively.

\section{Concepts of Swarm Intelligence}

\subsection{Basics of PSO}

Particle swarm optimization (PSO) is a population based optimization technique which is motivated by group manners of birds or fishes. Each particle has its own experience and neighbor particle experience. According to that particle adjusts its position, within 
the bounded area. Each particle has its local best (lbest) or a personal best. The best position brings into being by all neighbours particles in the specific solution is global best (gbest). The optimal solution is acquired in the specific solution in the course of its current velocity and the experience.

The formation of individual best is

$$
\text { Pbest }_{i}=\left(x_{i 1}^{\text {pbest }}, \cdots, x_{i n}^{\text {pbest }}\right)
$$

The pattern of global best is

$$
\text { Gbest }_{i}=\left(x_{i 1}^{\text {gbest }}, \cdots, x_{i n}^{\text {gbest }}\right)
$$

The velocity and the positions are updated in the each iteration. The updating of velocity is

$$
V_{i}^{k+1}=\omega V_{i}^{k}+c_{1} \text { rand }_{1} \times\left(\text { Pbest }_{i}^{k}-X_{i}^{k}\right)+c_{2} \text { rand }_{2} \times\left(\text { Gbest }_{i}^{k}-X_{i}^{k}\right)
$$

The updating of position of the particle is

$$
X_{i}^{k+1}=X_{i}^{k}+V_{i}^{k+1}
$$

$V_{i}^{k}$ current velocity of individual $i$ at iteration $k, V_{i}^{k+1}$ Modified velocity of individual $i$ at iteration $k+1, X_{i}^{k}$ Current position of individual $i$ at iteration, $\omega$ Inertia weight parameter, $c_{1}, c_{2}$ Acceleration factors, $\operatorname{rand}_{1}, \operatorname{rand}_{2}$ Random numbers between 0 and 1, Pbest $t_{i}^{k}$ Best position of individual $i$ until iteration $k$, Gbest ${ }_{i}^{k}$ Best position of the group until iteration $k$.

\subsection{Ant Colony Optimization}

Ant colony optimization (ACO) is an evolutionary and adaptive algorithm inspired by the behavior of real ant colonies. The ant when searching food and find food sources, it deposits a chemical called pheromone as a trail during the return path. Based on the quantity and quality of the food available the quantity of pheromone deposited. The pheromone trails allow the ants to identify the shortest path between the ant nest and the food source. Ant colony optimization algorithm updates pheromone values in order to update solutions during run time [19].

The pheromone trail updating equation is stated as

$$
T_{i j}(t+\Delta t)=(1-\alpha) T_{i j}(t)+\Delta T_{i j}
$$

$\alpha$ is the pheromone trails evaporation co-efficient, which lies between 0 and $1, \Delta T_{i j}$ is the increment of edge for the period $\Delta t$.

To enhance the tempo of search in the optimal power flow problem, the chemical communication between the insects will help. It is the signal of wasp which produces alarm pheromone to the inspiration of anxiety in the colony. This increases the speed of search in the problem area.

The chemical communication signal is expressed as

$$
A=(B / 2 D \Pi r) \text { comperr }(r / \sqrt{4 D t})
$$


$A$ is the chemical signal, $B$ is the emission rate, $D$ is the diffusion coefficient, $r$ is the radius of the active space, $t$ is the time from the beginning of emission, Comperr is the complementary error function.

\subsection{Hybrid Swarm Intelligence}

ACO not suitable for large optimization problems because it takes long time to search the result and premature convergence. To overcome of finding the best solution with large search space, combine with another algorithm (PSO) which has a better solution. In the hybrid PSO-ACO, the PSO algorithm is used for pheromone update of ACO. This improves the global exploration capabilities of ACO algorithm. This also improves convergence performance of ACO.

The velocity updating equation is expressed as

$$
\Delta T_{i j}=A+\sum_{k=1}^{n} \Delta T_{i j}^{k}+c_{1} \text { rand }_{1} \times \sum_{k=1}^{n} \Delta T_{i j}^{\text {local }_{k}}+c_{2} \text { rand }_{2} \times \Delta T_{i j}^{\text {global }}
$$

$A$ is the chemical communication signal, $\Delta T_{i j}^{k}$ is the increment of pheromone trails laid on the edge by ant between $t$ and $t+\Delta t, \Delta T_{i j}^{\text {local }_{k}}$ is the local update value of ant $k$, $\Delta T_{i j}^{\text {global }}$ is the global update value of edge.

\subsection{Fuzzy Approach}

In the proposed method, the aim is to achieve a set of solution of the multiobjective optimal power flow problem. In the optimal power flow problem with conflicting objectives the single optimal solution does not determine the real solution. The Pareto optimal approach, it is feasible to find the set of solution instead of the single optimal solution. From the set of solution, the best solution is obtained from the fuzzy approach. The equation for the fuzzy approach for best solution is expressed as

$$
\mu^{l}=\sum_{i=1}^{j} \mu_{i}^{l} / \sum_{l=1}^{x} \sum_{i=1}^{j} \mu_{i}^{l}
$$

$x$ total number of non-dominated solutions.

$\mu_{i}^{l}=0$ when the objective function is greater than the maximum value of ith objective function.

$\mu_{i}^{l}=1$ when the objective function is greater than the minimum value of ith objective function.

\subsection{Proposed Hybrid Algorithm for the Optimal Power Flow Problem}

Step 1: Read input data include equality and inequality constraints.

Step 2: Initialize the population.

Step 3: Calculate the objective function.

Step 4: Apply Pareto optimal method to get the set of solution.

Step 5: Update the velocity using hybrid velocity updating equation.

Step 6: Update the position using position updating equation.

Step 7: Check for stopping criteria. If the iteration reaches the maximum iteration 
stop else go to step 5 .

Step 8: Apply Pareto method and determine the non-dominated solution.

Step 9: Find the best solution using fuzzy approach.

\section{Simulation Results}

The proposed system has been tested to IEEE 30-bus test system. The 30-bus IEEE test system has 41 transmission lines, six generators and four transformers (T6-9, T6-10, T4-12 and T27-28). The transformer taps are set between 0.9 to 1.1. The proposed algorithm has the particles of 30 .

Case 1: Single objective optimization.

The proposed algorithms analyzed with the various values of acceleration factors $\left(c_{1}\right.$ and $c_{2}$ ) individually. This analysis assists to achieve the suitable value of the acceleration factor for the further process. This optimal power flow problem was simulated with the acceleration factor $\left(c_{1}\right)$ varied from 1 to 3 , using hybrid algorithm. The best value is obtained at $c_{1}>2.5$. Table 1 shows the best values of the cost, loss and VSI for $c_{1}=2.5$ are $\$ 801.7 / \mathrm{hr}, 5.21 \mathrm{MW}$ and 0.14 p.u. respectively. The hybrid algorithm also simulated with the various values of the acceleration factor $\left(c_{2}\right)$. The best value is obtained at $c_{2}>$ 2. Table 2 shows the best values of the cost, loss and VSI for $c_{2}=2$ are, $\$ 801.72 / \mathrm{hr}, 5.24$ MW and 0.13 p.u. respectively.

Table 3 shows the single objective optimized values with the control variables using the algorithms of PSO, ACO and Hybrid algorithm respectively. It shows that, the reliable generation cost of PSO, ACO and Hybrid are $\$ 802.15 / \mathrm{hr}, \$ 804.86 / \mathrm{hr}$ and $\$ 801.54 / \mathrm{hr}$ respectively. Figure 1 displays the generation cost convergence characteristics of a single objective optimization of IEEE 30 test bus system using PSO, ACO and Hybrid algorithm. Figure 2 indicates the reduction of transmission loss using PSO, ACO and Hybrid algorithms is 5.974 MW, 9.42 MW and 5.208 MW respectively. Figure 3 indicates the voltage stability of PSO, ACO and Hybrid algorithms are 0.1307 p.u., 0.142 p.u. and 0.13 p.u. respectively. These results show that, hybrid algorithm results are distributed.

Table 1. Best optimized values of different $C_{1}$ using hybrid algorithm.

\begin{tabular}{cccccc}
\hline Acceleration factor & 1 & 1.5 & 2 & 2.5 & 3 \\
\hline Cost (\$/hr) & 803.7 & 802.4 & 801.9 & 801.7 & 801.9 \\
Loss (MW) & 5.41 & 5.322 & 5.24 & 5.21 & 5.91 \\
VSI (p.u.) & 0.151 & 0.15 & 0.149 & 0.14 & 0.153 \\
\hline
\end{tabular}

Table 2. Best optimized values of different $C_{2}$ using hybrid algorithm.

\begin{tabular}{cccccc}
\hline Acceleration factor & 1 & 1.5 & 2 & 2.5 & 3 \\
\hline Cost $(\$ / \mathrm{hr})$ & 803.89 & 802.75 & 801.72 & 801.76 & 801.78 \\
Loss $(\mathrm{MW})$ & 5.319 & 5.27 & 5.24 & 5.25 & 5.254 \\
VSI (p.u) & 0.131 & 0.129 & 0.13 & 0.132 & 0.131 \\
\hline
\end{tabular}


Table 3. Single objective optimized values of cost, loss and VSI using PSO, ACO and Hybrid algorithms.

\begin{tabular}{|c|c|c|c|}
\hline & PSO & $\mathrm{ACO}$ & Hybrid \\
\hline $\mathrm{P}_{\mathrm{g} 1}(\mathrm{MW})$ & 177.132 & 176.901 & 176.7 \\
\hline $\mathrm{P}_{\mathrm{g} 2}(\mathrm{MW})$ & 48.12 & 49.932 & 48.89 \\
\hline $\mathrm{P}_{\mathrm{g} 3}(\mathrm{MW})$ & 21.356 & 21.125 & 21.4719 \\
\hline $\mathrm{P}_{\mathrm{g} 4}(\mathrm{MW})$ & 22.878 & 21.468 & 21.6423 \\
\hline $\mathrm{P}_{\mathrm{g} 5}(\mathrm{MW})$ & 10.121 & 10.116 & 12.0878 \\
\hline $\mathrm{P}_{\mathrm{g} 6}(\mathrm{MW})$ & 12 & 12 & 12.00 \\
\hline $\mathrm{V}_{\mathrm{g} 1}$ & 1.05 & 1.045 & 1.045 \\
\hline $\mathrm{V}_{\mathrm{g} 2}$ & 1.0442 & 1.043 & 1.043 \\
\hline $\mathrm{V}_{\mathrm{g} 3}$ & 1.446 & 0.998 & 0.998 \\
\hline $\mathrm{V}_{\mathrm{g} 4}$ & 1.0408 & 1.009 & 1.009 \\
\hline $\mathrm{V}_{\mathrm{g} 5}$ & 0.9601 & 1.014 & 1.014 \\
\hline $\mathrm{V}_{\mathrm{g} 6}$ & 1.05 & 1.047 & 1.047 \\
\hline $\mathrm{T}_{6-9}$, (p.u.) & 1.01 & 1.012 & 1.012 \\
\hline $\mathrm{T}_{6-10}$, (p.u.) & 0.99 & 0.971 & 0.971 \\
\hline $\mathrm{T}_{4-12}$, (p.u.) & 1.01 & 1.023 & 1.023 \\
\hline $\mathrm{T}_{27-28}$, (p.u.) & 1.02 & 1.014 & 1.014 \\
\hline Cost $(\$ / h r)$ & 802.15 & 804.86 & 801.54 \\
\hline Loss (MW) & 5.974 & 9.42 & 5.208 \\
\hline VSI (p.u) & 0.1307 & 0.142 & 0.13 \\
\hline
\end{tabular}

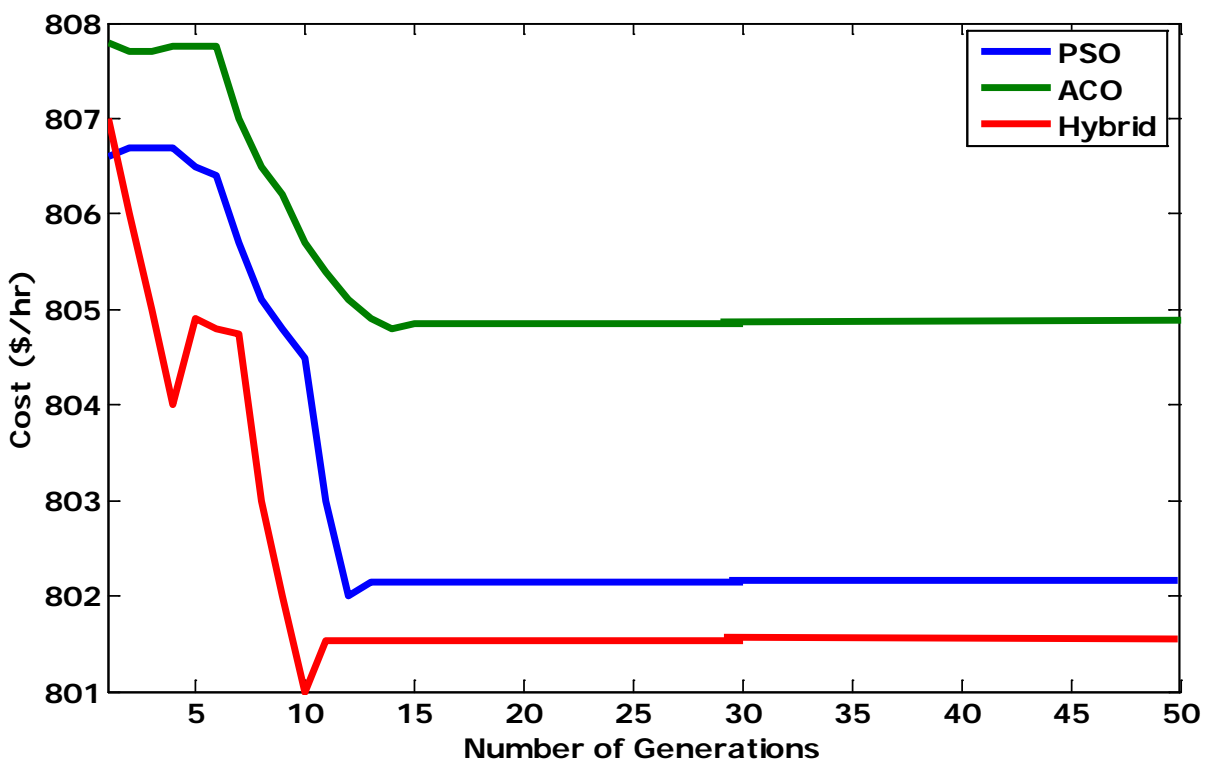

Figure 1. The generation cost convergence characteristics of a single objective optimization of IEEE 30 test bus system using PSO, ACO and hybrid algorithm. 
The accuracy of the proposed system is measured using the mean and standard deviation. Table 4 indicates the mean and standard values of the PSO, ACO and Hybrid algorithms. It is obvious that the proposed hybrid algorithm can achieve better result in the best solution search. The convergence time for the cost optimization using a hybrid technique is $1.05 \mathrm{~s}$, which is a faster than the PSO and ACO methods (1.24 s and $1.12 \mathrm{~s}$

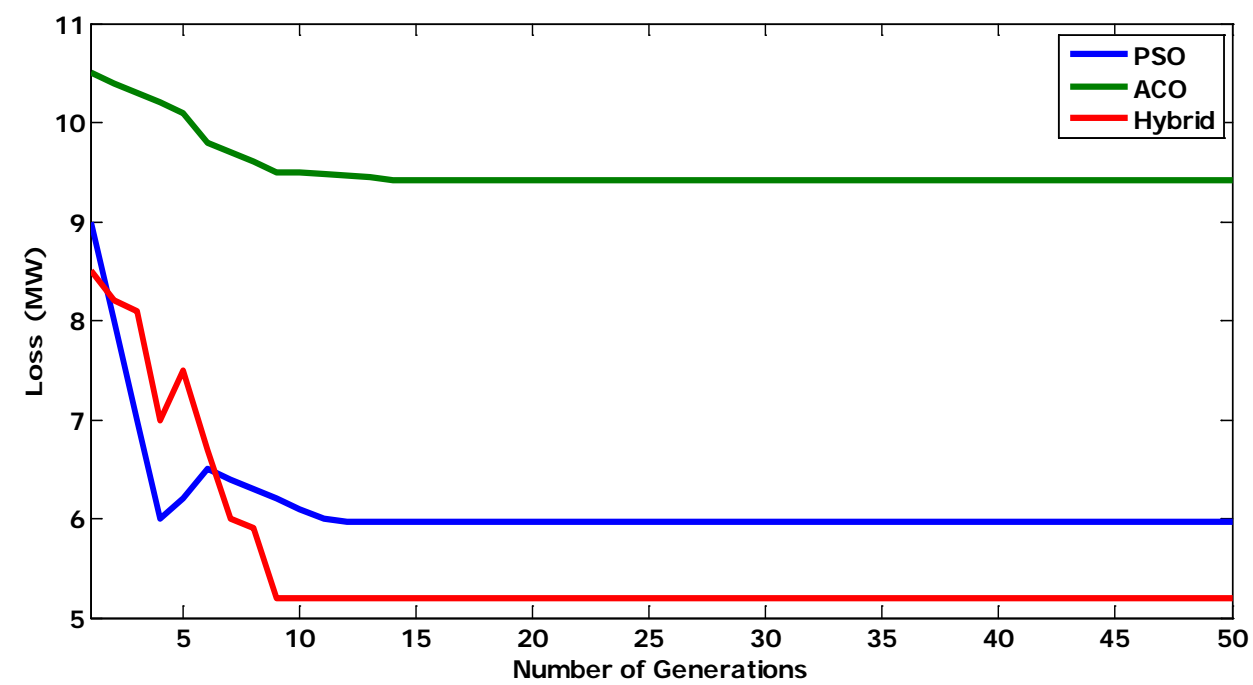

Figure 2. The transmission loss convergence characteristics of a single objective optimization of IEEE 30 test bus system using PSO, ACO and Hybrid algorithm.

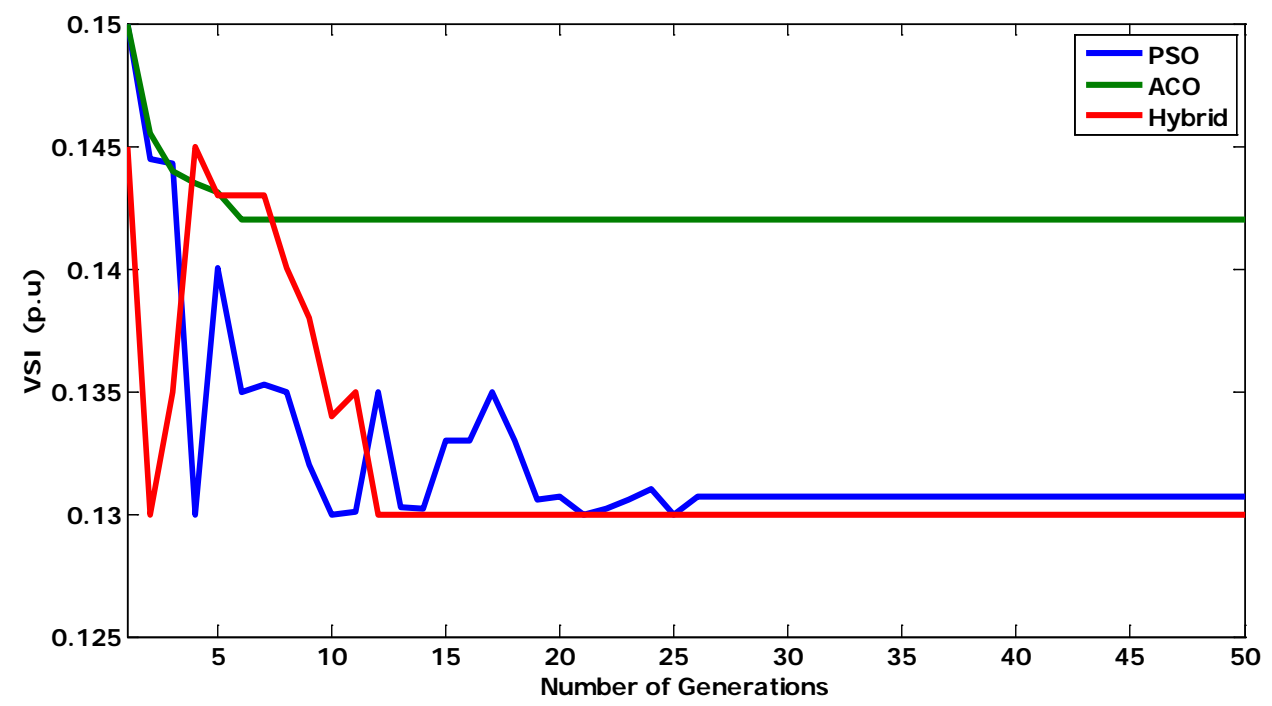

Figure 3. The voltage stability index convergence characteristics of a single objective optimization of IEEE 30 test bus system using PSO, ACO and hybrid algorithm.

Table 4. Performance measure of PSO, ACO and hybrid algorithms.

\begin{tabular}{cccc}
\hline & ACO & PSO & Hybrid \\
\hline Mean & 0.212 & 0.234 & 0.259 \\
STD & 0.042 & 0.036 & 0.024 \\
\hline
\end{tabular}


respectively). The convergence time taken for the hybrid technique is less. The accuracy and speed of convergence of hybrid are more than the ACO and PSO. Because the hybrid algorithm overcome the drawback in local search such as stagnation and premature convergence by the local experience of the ant and it processed in parallel. This enhances the chance of locating the finest solution in rapidity. The global search increased by the divergence of the pheromone tracks and also with the chemical communication signal. This leads the particle move the quickly to reach the solution. The global utilization, speed up the search and it support to acquire the best solution.

This optimal power flow problem was worked out using P4, $3 \mathrm{GHz}$. To evaluate the difficulty of the proposed system, the IEEE 14 bus is chosen to solve this OPF problem. The convergence time for this optimization in IEEE 14 bus system of hybrid method is $0.63 \mathrm{~s}$. The convergence time for the hybrid technique is for IEEE 30 bus test system is $1.05 \mathrm{~s}$ The convergence speed of the IEEE 30 bus system is twice the times of IEEE 14 bus test system. This will persuade to carry on the proposed technique in higher buses.

Case 2: Two objective optimization.

In a real world, the problems involve simultaneous optimization of several objective functions. These functions are non commensurable and conflicting objective functions. It gives rise to a set of optimal solutions, instead of one optimal solution. The reason for the optimality of many solutions is that no one can be considered to be better than any other with respect to all objective functions. Table 5 shows the optimized values of two

Table 5. Optimal solution for objective functions with different algorithms.

\begin{tabular}{|c|c|c|c|c|c|c|c|c|c|}
\hline & \multicolumn{3}{|c|}{ Cost-Loss } & \multicolumn{3}{|c|}{ Cost-VSI } & \multicolumn{3}{|c|}{ VSI-Loss } \\
\hline & PSO & $\mathrm{ACO}$ & Hybrid & PSO & $\mathrm{ACO}$ & Hybrid & PSO & $\mathrm{ACO}$ & Hybrid \\
\hline Pg1, MW & 136.467 & 132.374 & 134.717 & 178.167 & 179.232 & 178.12 & 177.458 & 172.486 & 169.451 \\
\hline Pg3, MW & 32.826 & 27.847 & 31.471 & 21.412 & 21.324 & 21.433 & 23.192 & 21.247 & 22.294 \\
\hline Pg4, MW & 21.686 & 32.694 & 30.642 & 16.234 & 17.415 & 16.574 & 17.497 & 19.782 & 18.482 \\
\hline $\mathrm{Vg} 1$ & 1.035 & 1.0265 & 1.045 & 1.049 & 1.056 & 1.045 & 1.049 & 1.056 & 1.045 \\
\hline Vg2 & 1.034 & 1.023 & 1.043 & 1.04 & 1.034 & 1.043 & 1.082 & 1.034 & 1.043 \\
\hline Vg3 & 0.998 & 0.948 & 0.998 & 0.890 & 0.998 & 0.998 & 1.089 & 0.998 & 0.998 \\
\hline $\mathrm{Vg} 4$ & 1.090 & 1.089 & 1.009 & 1.000 & 1.005 & 1.009 & 1.002 & 1.005 & 1.009 \\
\hline T6-10, pu & 0.9671 & 0.9598 & 0.971 & 0.980 & 0.992 & 0.971 & 0.980 & 0.992 & 0.971 \\
\hline $\mathrm{T} 4-12, \mathrm{pu}$ & 1.0123 & 1.002 & 1.023 & 1.034 & 1.039 & 1.023 & 1.034 & 1.039 & 1.023 \\
\hline $\mathrm{T} 27-28, \mathrm{pu}$ & 1.0124 & 1.0133 & 1.014 & 1.034 & 1.045 & 1.014 & 1.034 & 1.045 & 1.014 \\
\hline Cost $(\$ / \mathrm{hr})$ & 825.15 & 831.72 & 821.72 & 809.54 & 815.65 & 802.5 & & & \\
\hline Loss (MW) & 5.376 & 5.637 & 5.003 & & & & 5.2574 & 5.117 & 5.113 \\
\hline VSI (p.u) & & & & 0.1094 & 0.1096 & 0.1092 & 0.1047 & 0.1052 & 0.1007 \\
\hline Execution time (s) & 1.4815 & 1.675 & 1.4167 & 1.598 & 1.679 & 1.5297 & 1.4298 & 1.4972 & 1.4475 \\
\hline
\end{tabular}


objective optimization such as cost-loss, cost-VSI and loss-VSI using PSO, ACO and Hybrid algorithm. It is not easy to pinpoint the correct concert solution from a single run for the corresponding objective. So, to get the appropriate solution 30 numbers of pare to solution sets are obtained from 30 generations.

In the cost-loss minimization, objectives contrasts to each other. The cost-loss divergence optimization, the cost and loss for the PSO, ACO and Hybrid techniques are $\$ 825.15 / \mathrm{hr}, \$ 831.72 / \mathrm{hr}, \$ 821.72 / \mathrm{hr}$ and $5.376 \mathrm{MW}, 5.375 \mathrm{MW}, 5.043 \mathrm{MW}$ respectively. The reduction of cost and loss in hybrid is $1.12 \%$ and $11 \%$ higher than PSO and ACO techniques. Figure 4 indicates the compromised solution of cost and loss are $\$ 821.72 / \mathrm{hr}$ and 5.43 MW obtained using hybrid algorithm. It shows that, the cost-loss multiobjective optimization, the hybrid solution is best in cost and less reduction.

To avoid the stability problem in the system, the VSI should be maintained below critical limit. In addition to the VSI, cost also to be reduced. The cost-VSI conflict optimization, the cost and VSI for the PSO, ACO and Hybrid techniques are $\$ 809.54 / \mathrm{hr}$, $\$ 815.65 / \mathrm{hr}, \$ 802.5 / \mathrm{hr}$ and 0.1094 p.u, 0.1096 p.u., 0.1092 p.u, respectively. The reduction of cost and VSI of hybrid technique is $1.63 \%$ and $0.36 \%$ respectively. Figure 5 shows that the best compromised solution of cost and VSI are $\$ 802.5 / \mathrm{hr}$ and 0.1092 p.u, obtained using hybrid algorithm. The execution time of the hybrid technique for the cost-VSI divergence optimization is $1.5297 \mathrm{~s}$, which is a faster than the PSO and ACO methods (1.598 $\mathrm{s}$ and $1.679 \mathrm{~s}$ respectively).

In the loss-VSI case, the loss is minimized with the voltage stability, which is maintained below critical limit. The loss-VSI contrast optimization, the loss and VSI for the PSO, ACO and Hybrid techniques are 5.2574 MW, 5.117 MW, 5.113 MW and 0.1047 p.u, 0.1052 p.u., 0.1007 p.u, respectively. It shows that the reduction of loss in the ACO technique is less than the PSO technique Figure 6 indicates the divergence of hybrid is

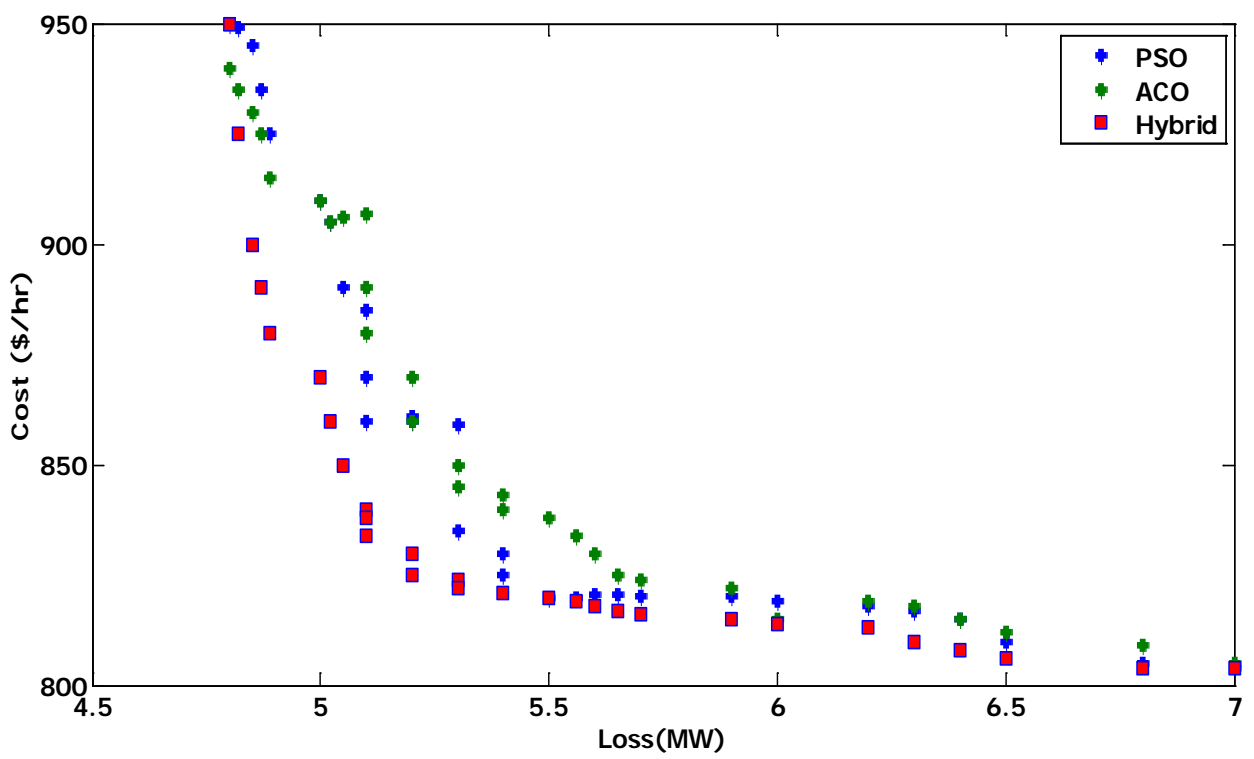

Figure 4. Loss-Cost optimization using PSO, ACO and hybrid algorithms of IEEE 30 test bus system. 


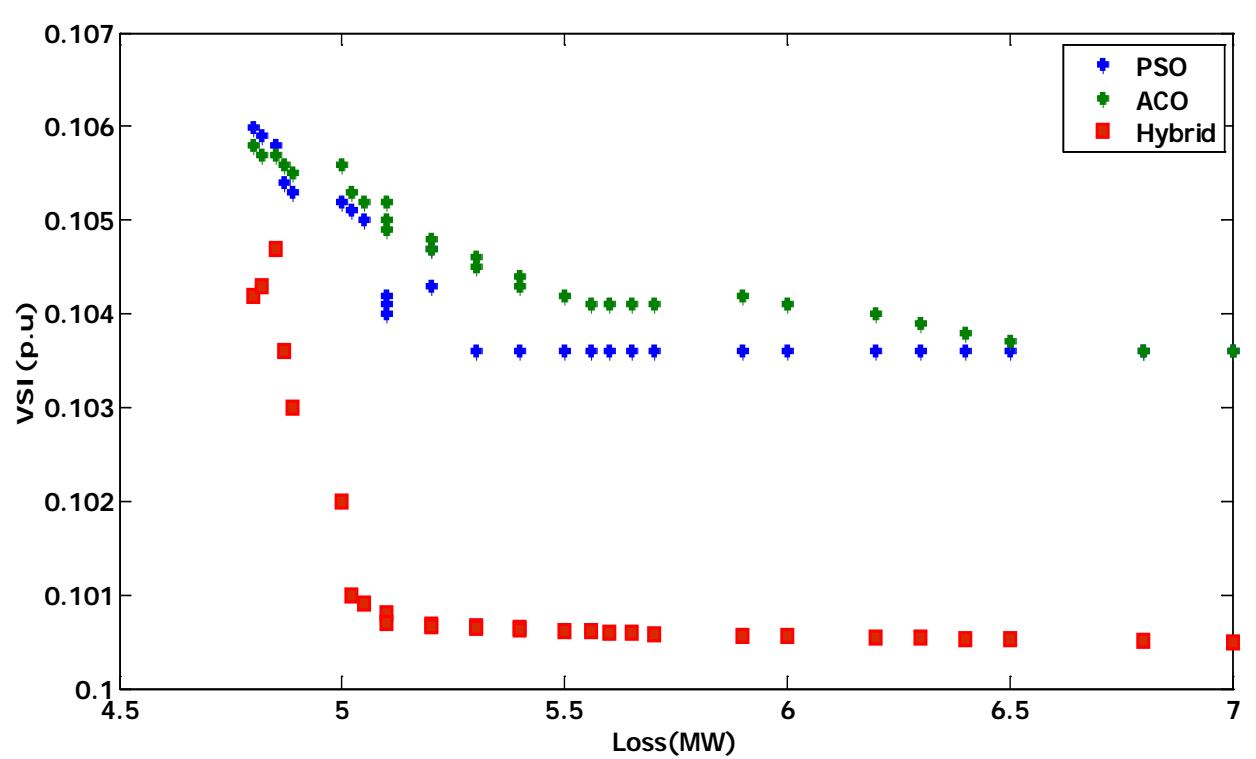

Figure 5. Loss-voltage stability index optimization using PSO, ACO and hybrid algorithm of IEEE 30 test bus system.

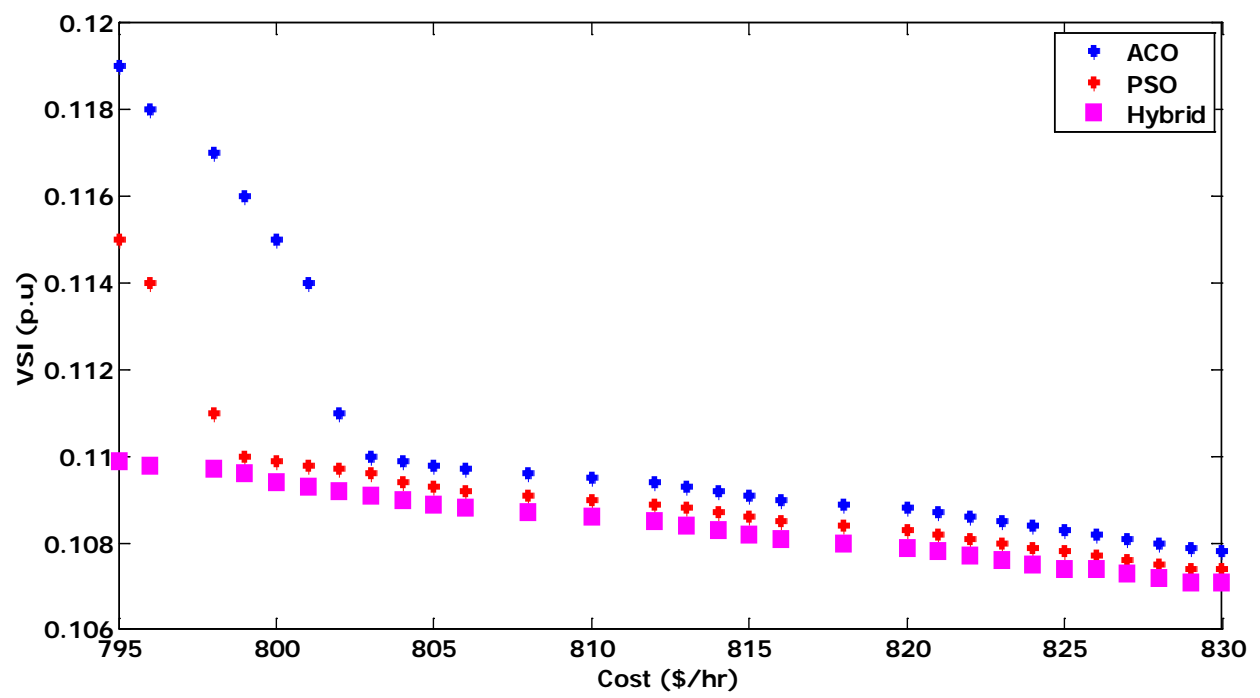

Figure 6. Voltage stability index-cost optimization using PSO, ACO and hybrid algorithm of IEEE 30 test bus system.

more than the ACO and PSO. The best compromised solution of loss and VSI are 5.113 MW and 0.1007 p.u, obtained using SPPSO algorithm. This result indicates the hybrid optimized result from the loss-VSI multiobjective optimization is better compared to the PSO and ACO techniques.

From the two objective optimization the reduction of cost is more in cost-VSI than the cost-loss optimization. The loss is optimized more in cost-loss multiobjective than the loss-VSI. Optimizing two objectives will give the dissimilar result for the same objective function. It initiates the objectives which are considered for the optimal power flow should be optimized simultaneously. 
Case 3: Three objective optimization.

Table 6 indicates the optimal values of the three objectives such as cost-loss-VSI, using PSO, ACO and Hybrid algorithms. The cost of the three objective optimization using PSO, ACO and Hybrid algorithms are $\$ 814.76 / \mathrm{hr}, \$ 815.6 / \mathrm{hr}$ and $\$ 805.5 / \mathrm{hr}$ respectively. In this, the cost reduced in the hybrid $1 \%$ more than the ACO and PSO techniques. The loss of the three objective optimization of the PSO, ACO and Hybrid techniques are 9.924 MW, 9.98 MW and 6.321 MW respectively. The loss reduction of hybrid technique is $57 \%$ higher than the PSO and ACO. The VSI of the PSO, ACO and Hybrid for the multi objective optimization are 0.1407 p.u, 0.1482 p.u and 0.1127 p.u respectively. The hybrid algorithm results, $24 \%$ more reduction in VSI than the PSO and ACO techniques. Table 6 shows, the hybrid technique results the better accuracy compare to the PSO and ACO algorithm for multiobjective optimization problem.

In order to confirm the performance of the proposed algorithm in large systems, hybrid algorithm applied in IEEE 118 test bus system. The particles are increased to 50 . To get the appropriate solution, 30 numbers of pareto solution sets are obtained from 30 generations. Table 7 indicates the optimal solutions for the cost, loss and VSI optimized with a single objective, two objectives and three objective function using a hybrid

Table 6. Multiobjective optimal solution for Cost-Loss-Voltage Stability Index with different algorithms.

\begin{tabular}{cccc}
\hline Variables & PSO & ACO & Hybrid \\
\hline $\mathrm{P}_{\mathrm{g} 1}, \mathrm{MW}$ & 168.163 & 167.937 & 105.71 \\
$\mathrm{P}_{\mathrm{g} 2}, \mathrm{MW}$ & 46.232 & 45.827 & 74.445 \\
$\mathrm{P}_{\mathrm{g} 3}, \mathrm{MW}$ & 23.957 & 23.211 & 38.329 \\
$\mathrm{P}_{\mathrm{g} 4}, \mathrm{MW}$ & 15.328 & 18.185 & 35.517 \\
$\mathrm{P}_{\mathrm{g} 5}, \mathrm{MW}$ & 18.541 & 17.248 & 19.278 \\
$\mathrm{P}_{\mathrm{g} 6}, \mathrm{MW}$ & 23.789 & 25.157 & 12.248 \\
$\mathrm{~V}_{\mathrm{g} 1}$ & 1.065 & 1.074 & 1.045 \\
$\mathrm{~V}_{\mathrm{g} 2}$ & 1.054 & 1.063 & 1.043 \\
$\mathrm{~V}_{\mathrm{g} 3}$ & 0.9002 & 0.9098 & 0.998 \\
$\mathrm{~V}_{\mathrm{g} 4}$ & 1.100 & 1.104 & 1.009 \\
$\mathrm{~V}_{\mathrm{g} 5}$ & 1.017 & 1.019 & 1.014 \\
$\mathrm{~V}_{\mathrm{g} 6}$ & 1.066 & 1.078 & 1.047 \\
$\mathrm{~T}_{6-9}, \mathrm{pu}$ & 1.015 & 1.019 & 1.012 \\
$\mathrm{~T}_{6-10}, \mathrm{pu}$ & 0.980 & 0.998 & 0.971 \\
$\mathrm{~T}_{4-12}, \mathrm{pu}$ & 1.034 & 1.045 & 1.023 \\
$\mathrm{~T}_{27-28}, \mathrm{pu}$ & 1.016 & 1.017 & 1.014 \\
Cost $(\$ / \mathrm{hr})$ & 814.76 & 815.6 & 8.321 \\
Loss $\left._{2} \mathrm{MW}\right)$ & 9.924 & 9.98 & 1.5348 \\
$\mathrm{VSSI}_{\mathrm{E}}(\mathrm{p} . \mathrm{u})$ & 0.1407 & 0.1482 &
\end{tabular}


Table 7. The optimal solution of single objective and multi objective functions using hybrid algorithm for IEEE 118 test bus system.

\begin{tabular}{cccccccc}
\hline \multirow{2}{*}{ Objectives } & \multicolumn{3}{c}{ Single objective } & \multicolumn{3}{c}{ Two objectives } & Three objectives \\
\cline { 2 - 7 } & Cost & Loss & VSI & Cost-loss & Cost-VSI & Loss-VSI & Cost-loss-VSI \\
\hline Cost (\$/hr) & 128945 & 152014 & 149156 & 131992 & 131129 & & 131119 \\
Loss (MW) & 139.5 & 123.3 & 138.6 & 115.4 & & 125.12 & 143.5 \\
VSI (p.u.) & 0.47 & 0.479 & 0.412 & & 0.431 & 0.429 & 0.442 \\
Execution time (s) & & & & 35.487 & 35.897 & 35.127 & 48.087 \\
\hline
\end{tabular}

algorithm for IEEE 118 test bus system. It indicates the values of cost, loss and VSI of the single objective optimization are less when compared to the multiobjective optimization. It states that while considering more objective function, the optimized value and execution time are increased concurrently. Because of nonlinearity in large system the front is less consistent comparing to the smaller system.

\section{Conclusion}

In this paper optimal power flow problem has three objectives with constraints, and it works out in the multiobjective optimization approach. This multiobjective problem is solved by single objective, two-objective optimization such as cost-loss, cost-voltage stability index, loss-voltage stability index, and three-objective optimization of cost-lossvoltage stability index using PSO, ACO and hybrid techniques. Hybrid swarm intelligence is used to avoid the stagnation of global and local search and get the best solution by fuzzy approach. The simulation results show that the hybrid swarm optimization provides better results and faster convergence compared to the ant colony optimization and particle swarm optimization. The proposed technique gives better results with less convergence time and it will help to choose the sensible solution to the multiobjective optimization problem in IEEE 30 \& IEEE 118 test bus system.

\section{References}

[1] Burchet, R.C., Happ, H.H. and Vierath, D.R. (1984) Quadratically Convergent Optimal Power Flow. IEEE Transactions on Power Apparatus and Systems, 103, 3267-3276. http://dx.doi.org/10.1109/TPAS.1984.318568

[2] Capitanescu, F., Glavic, M., Ernst, D. and Wehenkel, L. (2007) Interior-Point Based Algorithms for the Solution of Optimal Power Flow Problems. Electric Power Systems Research, 77, 508-517. http://dx.doi.org/10.1016/j.epsr.2006.05.003

[3] Olofsson, M., Anderson, G. and Soder, L. (1995) Linear Programming Based Optimal Power Flow Using Second Order Sensitivities. IEEE Transactions on Power Systems, 10, 16911697. http://dx.doi.org/10.1109/59.466472

[4] Salgado, R., Brameller, A. and Aitchison, P. (1990) Optimal Power Flow Solutions Using the Gradient Projection Method Part 2: Modelling of the Power System Equations, Generation, Transmission and Distribution. IEE Proceedings, 137, 429-435.

[5] Sun, D.I., Ashley, B., Brewer, B., Hughes, A. and Tinney, W.F. (1984) Optimal Power Flow by Newton Approach. IEEE Transaction on Power Apparatus and Systems, PAS-103, 2864- 
2880. http://dx.doi.org/10.1109/TPAS.1984.318284

[6] Abido, M.A. (2002) Optimal Power Flow Using Particle Swarm Optimization. Electrical Power \& Energy System, 24, 563-571. http://dx.doi.org/10.1016/S0142-0615(01)00067-9

[7] Todorovski, M. and Rajicic, D. (2006) An Initialization Procedure in Solving Optimal Power Flow by Genetic Algorithm. IEEE Transactions on Power Systems, 21, 480-487. http://dx.doi.org/10.1109/TPWRS.2006.873120

[8] Bakirtzis, A.G., Biskas, P.N., Zoumas, C.E. and Petridis, V. (2002) Optimal Power Flow by Enhanced Genetic Algorithm. IEEE Transactions on Power Systems, 17, 229-236. http://dx.doi.org/10.1109/TPWRS.2002.1007886

[9] Yuryevich, J. and Wong, K.P. (1999) Evolutionary Programming Based Optimal Power Flow Algorithm. IEEE Transactions on Power Systems, 14, 1245-1250. http://dx.doi.org/10.1109/59.801880

[10] Abido, M.A. (2002) Optimal Power Flow Using Tabu Search Algorithm. Electric Power Components and Systems, 30, 469-483. http://dx.doi.org/10.1080/15325000252888425

[11] Varadarajan, M. and Swarup, K.S. (2008) Solving Multi-Objective Optimal Power Flow Using Differential Evolution. IET Generation, Transmission \& Distribution, 2, 720-730. http://dx.doi.org/10.1049/iet-gtd:20070457

[12] Abou El Elaa, A.A., Abidob, M.A. and Speaa, S.R. (2010) Optimal Power Flow Using Differential Evolution Algorithm. Electric Power Systems Research, 80, 878-885.

http://dx.doi.org/10.1016/j.epsr.2009.12.018

[13] Kumari, M.S. and Maheswarapu, S. (2010) Enhanced Genetic Algorithm Based Computation Technique for Multi-Objective Optimal Power Flow Solution. Electrical Power and Energy Systems, 32, 736-742. http://dx.doi.org/10.1016/j.ijepes.2010.01.010

[14] Shi, L.B. and Xu, G.Y. (2001) Self-Adaptive Evolutionary Programming and Its Application to Multi-Objective Optimal Operation of Power Systems. Electric Power Systems Research, 57, 181-187. http://dx.doi.org/10.1016/S0378-7796(01)00086-4

[15] Niknam, T., Narimani, M.R., Aghaei, J. and Azizipanah-Abarghooee, R. (2012) Improved Particle Swarm Optimisation for Multi-Objective Optimal Power Flow Considering the Cost, Loss, Emission and Voltage Stability Index. IET Generation, Transmission \& Distribution, 6, 515-527. http://dx.doi.org/10.1049/iet-gtd.2011.0851

[16] Sivasubramani, S. and Swarup, K.S. (2011) Multi-Objective Harmony Search Algorithm for Optimal Power Flow Problem. Electrical Power and Energy Systems, 33, 745-752. http://dx.doi.org/10.1016/j.ijepes.2010.12.031

[17] Fonseca, C.M. and Fleming, P.J. (1995) An Overview of Evolutionary Algorithms in Multiobjective Optimization. Evolutionary Computation, 3, 1-16. http://dx.doi.org/10.1162/evco.1995.3.1.1

[18] Rosehart, W.D., Cañizares, C.A. and Quintana, V.H. (2003) Multiobjective Optimal Power Flows to Evaluate Voltage Security Costs in Power Networks. IEEE Transactions on Power Systems, 18, 578-587. http://dx.doi.org/10.1109/TPWRS.2003.810895

[19] Shuang, B., Chen, J.P. and Li, Z.B. (2011) Study on Hybrid PS-ACO Algorithm. Application Intelligence, 34, 64-73. http://dx.doi.org/10.1007/s10489-009-0179-6 
Submit or recommend next manuscript to SCIRP and we will provide best service for you:

Accepting pre-submission inquiries through Email, Facebook, LinkedIn, Twitter, etc. A wide selection of journals (inclusive of 9 subjects, more than 200 journals)

Providing 24-hour high-quality service

User-friendly online submission system

Fair and swift peer-review system

Efficient typesetting and proofreading procedure

Display of the result of downloads and visits, as well as the number of cited articles

Maximum dissemination of your research work

Submit your manuscript at: http://papersubmission.scirp.org/ 